\title{
Erratum: An array of options: proteomics gets parallel
}

Alan Dove

Nat. Methods 2, 709 (2005).

As illustration on page 712, an image of the Biacore X model was mistakenly used instead of an image of the Biacore T100 model.

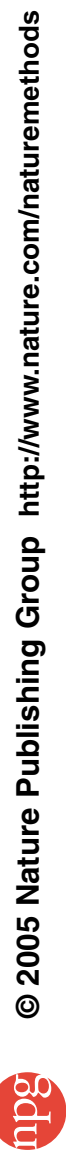

